\title{
Deterministic Optimization and Reliability Analysis of a Cam Mechanism with Translating Flat-Face Follower
}

\author{
A. Redjechta ${ }^{1, *}$, F. Djeddou ${ }^{2}$, H. Ferhat ${ }^{2}$ \\ ${ }^{1}$ Emerging Materials Research Unit, Ferhat Abbas-Setif University 1, Algeria \\ ${ }^{2}$ Laboratory of Applied Precision Mechanics, Ferhat Abbas-Setif University 1, Algeria
}

Received August 15, 2019; Revised September 20, 2019; Accepted September 27, 2019

Copyright $\bigcirc 2019$ by authors, all rights reserved. Authors agree that this article remains permanently open access under the terms of the Creative Commons Attribution License 4.0 International License

\begin{abstract}
The objective of this work is to perform a synthesis of a cam mechanism of the first species, with translating flat-face follower, based on deterministic and probabilistic approaches. The study consists of two parts: the first consists of optimization of the mechanism to obtain an optimal size and ensure a more efficient operation, taking into account the three major parameters that generally influence the design of this type mechanism, namely the base radius of the cam, the length of the plate and the eccentricity of the rod. In addition, the constraints related to the performance and resistance indicators as well as the geometric conditions are taken into account. The second part of this work is devoted to the reliability analysis whose probability of system failure is estimated by the approximate methods: FORM / SORM and Monte Carlo simulation, using the software Phimeca Software. Sensitivity measurements have demonstrated their effectiveness on system reliability by making it more robust.
\end{abstract}

Keywords Cam Mechanism, Optimization, Reliability, FORM Method, SORM Method

\section{Introduction}

Cam-rod mechanisms are considered among the most prevalent and important systems that are found today in modern machines. Their applications are found almost in all devices and mechanical machines including those of the automatic type, such as printing presses, shoe machines, textile machines, machining machines, etc. Since a large cam size implies large inertia forces, this can lead to high stresses between the cam and the rod [1]. However, the common practice in the design of cam mechanisms is to look for the optimal size of the cam with the aim of making the operation more consistent.
The optimal design of cam mechanisms is dealt with in many publications [2-5], where various constraints and methods are considered. A multi-objective optimization approach of an eccentric cam mechanism was developed in reference [6], the proposed objective function aims at minimizing the overall size of the system and maximizing efficiency. In reference [7], the design parameters of a roller-ended cam mechanism were determined by minimizing the maximum pressure stress at the contact between the cam and the roller. Bouzakis et al. [8] developed an algorithm for an optimal synthesis of a second-type cam mechanism. Terauchi and El-Shakery [9] developed an algorithm based on iterative numerical methods to optimize the size of the cam by respecting pressure constraints.

On the other hand, very little work has been done on the reliability of cam mechanisms [10, 11], although reliability-based design methods are becoming widely used to obtain robust designs in engineering. In [10], the effect of manufacturing tolerances of the cam profile on the output movement was studied. In this work, the mathematical expression of the cam profile was derived from the desired displacement function, and then the variance due to the cut tolerances was modeled using Monte Carlo simulation. A computerized approach to the tolerances of a roller cam mechanism has been developed in [11] using the concept of simulated upper pair contact analysis. In this work, the kinematic deviation of rod motion resulting from the tolerance quantity of each design parameter was determined numerically.

The purpose of this paper is to synthesize a plate-like, first-order cam mechanism based on deterministic approaches and reliability analysis. The study consists of two parts: the first part performs a preliminary deterministic optimization in order to obtain an optimal size of the system and to ensure a more efficient operation. For this purpose, an objective function is defined which takes into account the three main parameters which 
generally influence the design of this type of mechanism, namely the basic radius of the cam $R_{b}$, the length of the plate and the eccentricity of the rod, while respecting the constraints related to the performance and resistance indicators such as the pressure angle, the radius of curvature of the cam and the contact pressure, as well as geometric conditions. The second part is devoted to a reliability study whose probability of system failure is estimated by two complementary methods: the FORM / SORM approximation methods and the Monte Carlo simulation using the Phimeca reliability software [12].

\section{Objective Function and Constraints}

The objective function defined in this study aims to minimize the size and maximize the performance of the mechanism. The size is presented by two parameters, the base radius of the cam $R_{b}$ and the length of the plate $L$. While the performance of the mechanism is related to the reduction of the moment, and the maximum contact pressure applied to the cam. The design variables are therefore the base radius $R_{b}$, the length of the plate, $L$, and the eccentricity of the rod, e (Fig.1).

The objective function is defined as follows:

$$
f\left(R_{b}, L, e\right)=\beta_{1} \cdot\left(R_{b}+L\right)+\beta_{2} \cdot T_{\max }+\beta_{3} \cdot \sigma_{\max }
$$

In order to ensure the proper functioning of the mechanism, the first constraint is imposed on the maximum pressure angle and is fixed as follows [13]:

$$
g_{1}=30^{\circ} \geq \varphi_{\max }
$$

The pressure angle is evaluated using the known law of motion (see Figure 2)



Figure 1. Kinematic diagram of a cam mechanism terminated by a plate [14] 

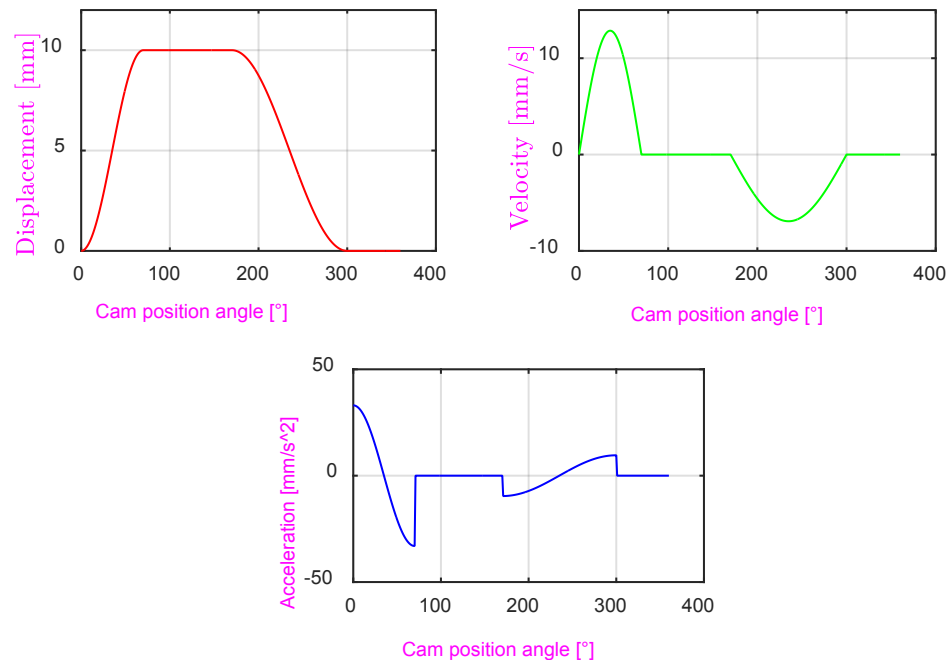

Figure 2. a). Displacement diagram, b) Analogous velocity diagram, and c) Analogous acceleration diagram

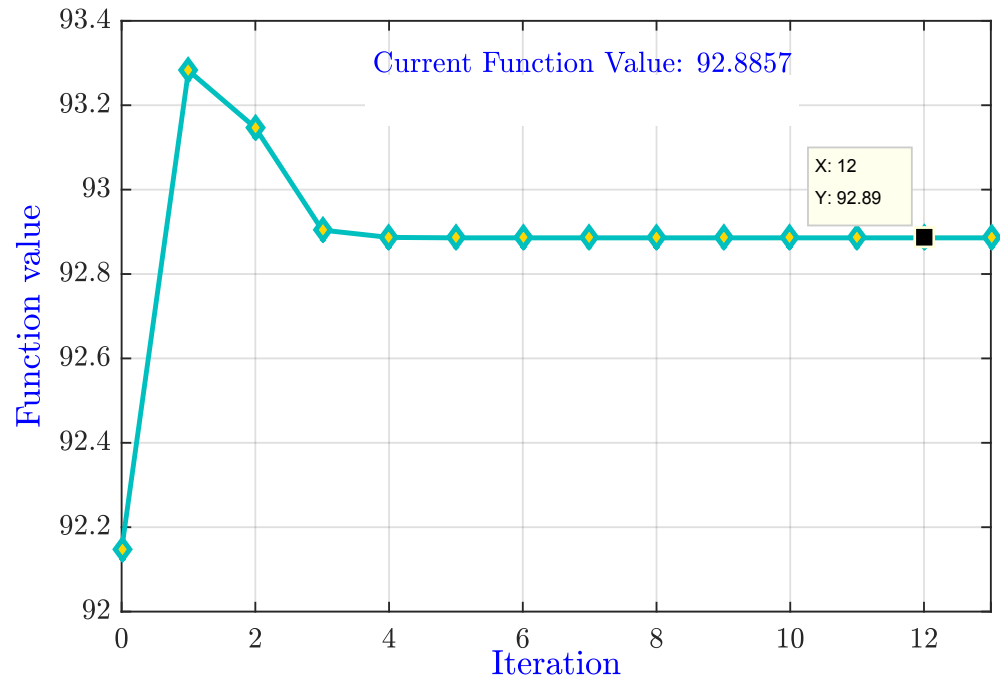

Figure 3. Evolution of the objective function according to iterations (fmincon)

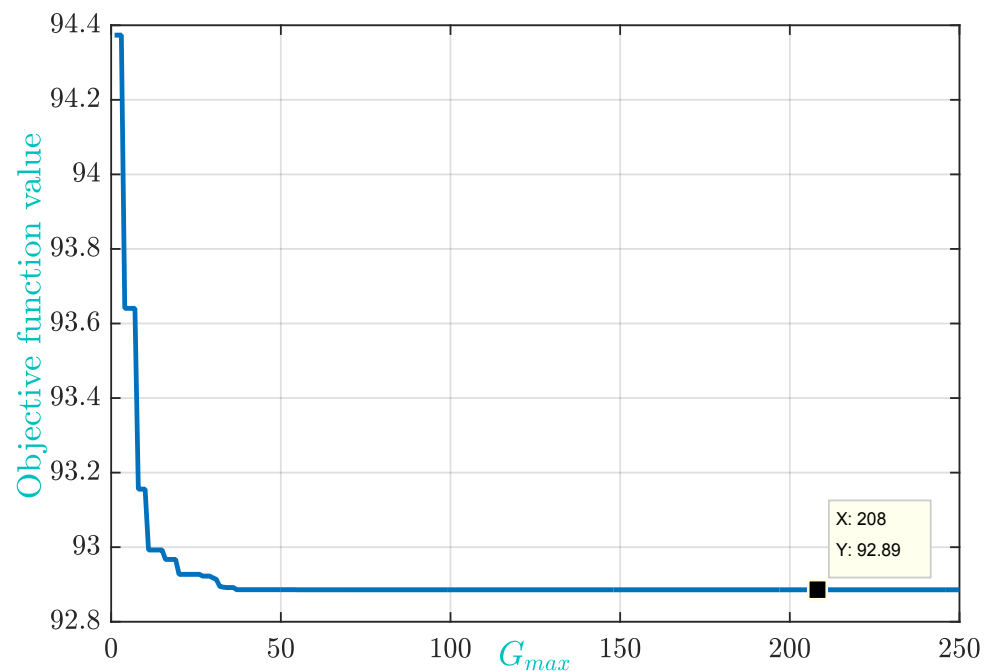

Figure 4. Evolution of the objective function according to the number of generations (DE) 
The second constraint is fixed to ensure the resistance of the cam.

$$
g_{2}=\sigma_{a d m} \geq \sigma_{\max }
$$

with $\sigma_{\max }$ is the maximal applied contact stress and $\sigma_{a d m}$ is the permissible contact pressure.

The third constraint stems from Fig.1 [14].

$$
g_{3}=\frac{L}{2} \geq \alpha
$$

with $\alpha$ given by the following relation [15]:

$$
\alpha=\sqrt{\left(x^{2}+y^{2}\right)-\left(R_{b}+s\right)^{2}}
$$

Where $x, y$ are the coordinates of the profile of the cam, and $S$ the displacement of the rod.

To avoid jamming the mechanism, the fourth constraint is fixed as follows [15]:

$$
g_{4}=b-2 \alpha \mu+b \mu \mu_{0}+(1-2 \xi)>0
$$

with $\mu_{0}$ the coefficient of friction between the cam and the plate, $\mu$ the coefficient of friction between the rod and its guide, $\mathrm{b}$ and $\xi$ are presented in Fig.1

The fifth constraint, imposed on the radius of curvature, is given by [16]:

$$
g_{5}=\rho_{\min } \geq 0
$$

The radius of curvature of the cam is evaluated using the known law of motion (see Figure 2).

The sixth constraint is a geometric condition [15]:

$$
g_{6}=\frac{L}{2} \geq e
$$

The lower and upper bounds of the variables must be defined:

$$
\begin{gathered}
20 \mathrm{~mm} \leq R_{b} \leq 60 \mathrm{~mm} \\
20 \mathrm{~mm} \leq L \leq 40 \mathrm{~mm} \\
0 \mathrm{~mm} \leq e \leq 0 \mathrm{~mm}
\end{gathered}
$$

\section{Presentation of Optimization Results}

In this section, an example is presented and the results are illustrated. The Inputs data of the studied example are tabulated in table 1 [17]. The rod follows a cosine law of the type mounted-stop-back-stop, the input parameters are:

The rise of the stem: $\mathrm{h}=10 \mathrm{~mm}$.

The rise angle: $\theta_{m}=70^{\circ}$.

The stopping angle at the top: $\theta_{r}=100^{\circ}$.

The descent angle: $\theta_{d}=130^{\circ}$.

The stopping angle at the bottom: $\theta_{b}=60^{\circ}$.
Table 1. Inputs from optimization

\begin{tabular}{|c|c|}
\hline Parameters & Value \\
\hline External load $\mathrm{F}_{\mathrm{e}}(\mathrm{N})$ & 30 \\
\hline stiffness Constant $k\left(\frac{\mathrm{N}}{\mathrm{mm}}\right)$ & 1.2 \\
\hline Mass of the rod $M(\mathrm{Kg})$ & 0.05 \\
\hline Rotation speed $\omega_{c}\left(\frac{\mathrm{rad}}{\mathrm{sec}}\right)$ & 2 \\
\hline$\mu_{0}$ & 0.15 \\
\hline$\mu$ & 0.1 \\
\hline$\sigma_{a d m}(\mathrm{Mpa})$ & 235 \\
\hline $\mathrm{b}(\mathrm{mm})$ & 60 \\
\hline
\end{tabular}

Figures 2a-c respectively shows the displacement, velocity and acceleration diagrams of the rod for the law of the considered movement.

To solve this problem, the fmincon subroutine of MATLAB is used. This algorithm, which implements a quasi-Newtonian minimization routine with constraints, generally exhibits good results in solving technology problems. In addition, the results obtained by fmincon are validated by an evolutionary algorithm, namely, Adaptive Mixed Differential Evolution (FMEA) [18]. The control parameters considered by AMDE for this study are as follows:

The size of the population is NP $=30$.

The maximum number of generations is $\mathrm{Gmax}=250$.

The mutation factors are $\mathrm{F} 1=0.6, \mathrm{~F} 2=0.8$.

The probability of crossing $\mathrm{CR}=0.95$.

Table 2 shows the optimization results obtained by the two methods used. The weight factors are chosen as follows. The evaluation of the objective function for the optimization case by the fmincon function and DE for the example study is shown respectively in Figure 3 and Figure 4.

Table 2. Optimization results

\begin{tabular}{|c|c|c|}
\hline Settings & Fmincon & AMDE \\
\hline$R_{b}(\mathrm{~mm})$ & 32.8399 & 32.8399 \\
\hline$L(m m)$ & 25.7143 & 25.7143 \\
\hline$e(m m)$ & 9.9405 & 9.9405 \\
\hline$g_{1}$ & -4.5690 & -4.5690 \\
\hline$g_{2}$ & -83.609 & -83.609 \\
\hline$g_{3}$ & -47.209 & -47.209 \\
\hline$g_{4}$ & -0.0000 & -0.0000 \\
\hline$g_{5}$ & -9.7786 & -9.7786 \\
\hline$g_{6}$ & -2.9166 & -2.9166 \\
\hline$f$ & 92.8857 & 92.8857 \\
\hline
\end{tabular}

From both figures 3 and 4 and the results of table 2, it is clear that both methods converge towards the same results, where the minimum value of the objective function is 92.8857 for $\mathrm{x} *=[32.8399$ 25.7143 9.9405]. Moreover, we find that the optimum is reached with a total satisfaction of the constraints for the two methods. After obtaining the results of the optimization, the optimal profile of the cam is presented in Fig. 5. 


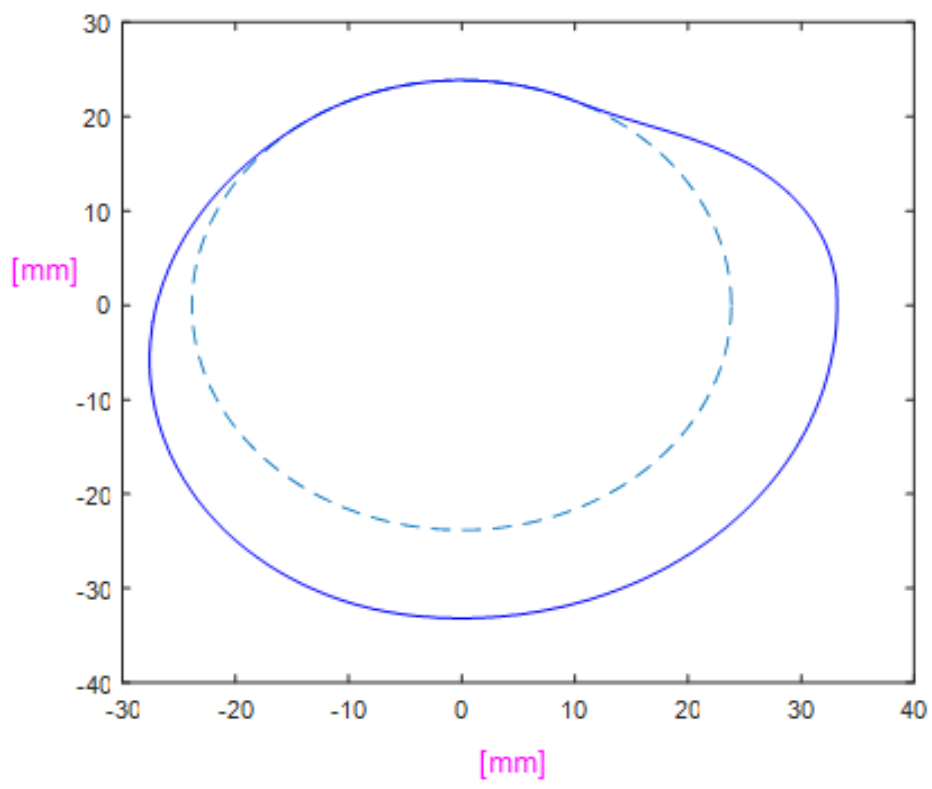

Figure 5. Profile of the optimal cam obtained

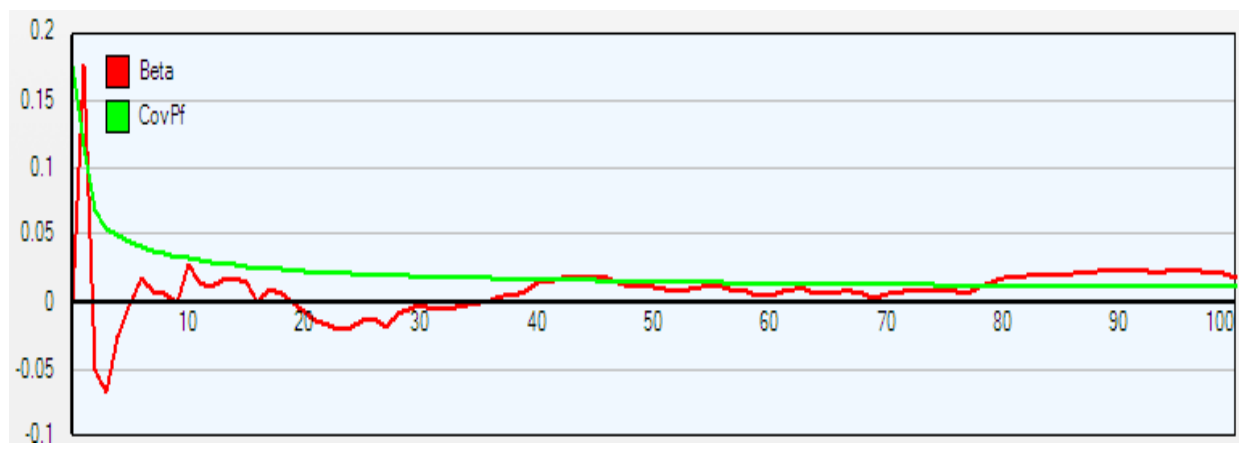

Figure 6. Convergence graph obtained by the Monte Carlo simulation

\section{Reliability Analyses}

This part is devoted to the reliability analysis of our mechanism; the uncertainty is taken into account, whose Pf failure probability of the system is estimated by two complementary methods: the FORM/SORM approximation methods and the Monte Carlo simulation using the reliable software Phimeca Software. A sensitivity study is then performed to determine the weight of each parameter on the performance of the system. The performance function of the system treats the third constraint and is written as follows:

$$
G(X)=\frac{L}{2}-\alpha
$$

The term resistance is therefore the length of the plateau, and the term solicitation is the distance $\alpha$. The failure is observed when $G<0$. The three design variables of this mechanism are taken as random variables according to the normal distribution and its statistical data are shown in Table 3. It is noted that all the tolerances of the variables are taken according to the bibliographic source [19] and are independent.

Table 3. Random variables of the system

\begin{tabular}{|c|c|c|c|}
\hline $\begin{array}{c}\text { Random } \\
\text { Variable }\end{array}$ & Mean & Law & $\begin{array}{c}\text { Coefficient of variation } \\
(\%)\end{array}$ \\
\hline$R_{b}(\mathrm{~mm})$ & 32.8399 & Normal & 0.5 \\
\hline$L(\mathrm{~mm})$ & 25.7143 & Normal & 0.5 \\
\hline$e(\mathrm{~mm})$ & 09.9405 & Normal & 0.5 \\
\hline
\end{tabular}

\subsection{Reliability Results}

Table 4. Reliability results.

\begin{tabular}{|c|c|c|c|}
\hline Method & Call Number & $P_{f}$ & $\beta\left(10^{-4}\right)$ \\
\hline FORM (Abdo-Rackwitz) & 120 & 0.4997 & 7.78 \\
\hline SORM (Abdo-Rackwitz) & 125 & 0.4997 & 7.78 \\
\hline Monte Carlo Simulation & 100 & 0.4927 & 182.99 \\
\hline
\end{tabular}

The results obtained by the FORM / SORM methods and the Monte Carlo simulation is shown in the following table. The latter is taken as a reference solution with 100 simulations. 
By analyzing Table 4, it is observed that the probability of system failure is high. This has a negative effect on the performance of the mechanism.

\subsection{Sensitivity Analysis}

Figure 7 and 8 respectively show the importance factors and the diagrams of the elasticities coming from the
Phimeca software of the different parameters of this study. The elasticity of a parameter (mean, standard deviation) is a dimensional indicator of the relative weight of each parameter on the evaluation of Pf. The interpretation of the importance factors, Fig.7, shows that the average of the length $L$ of the plateau has a great influence $(76.21 \%)$ on the evolution of the reliability index $\beta$ compared to the other variables.

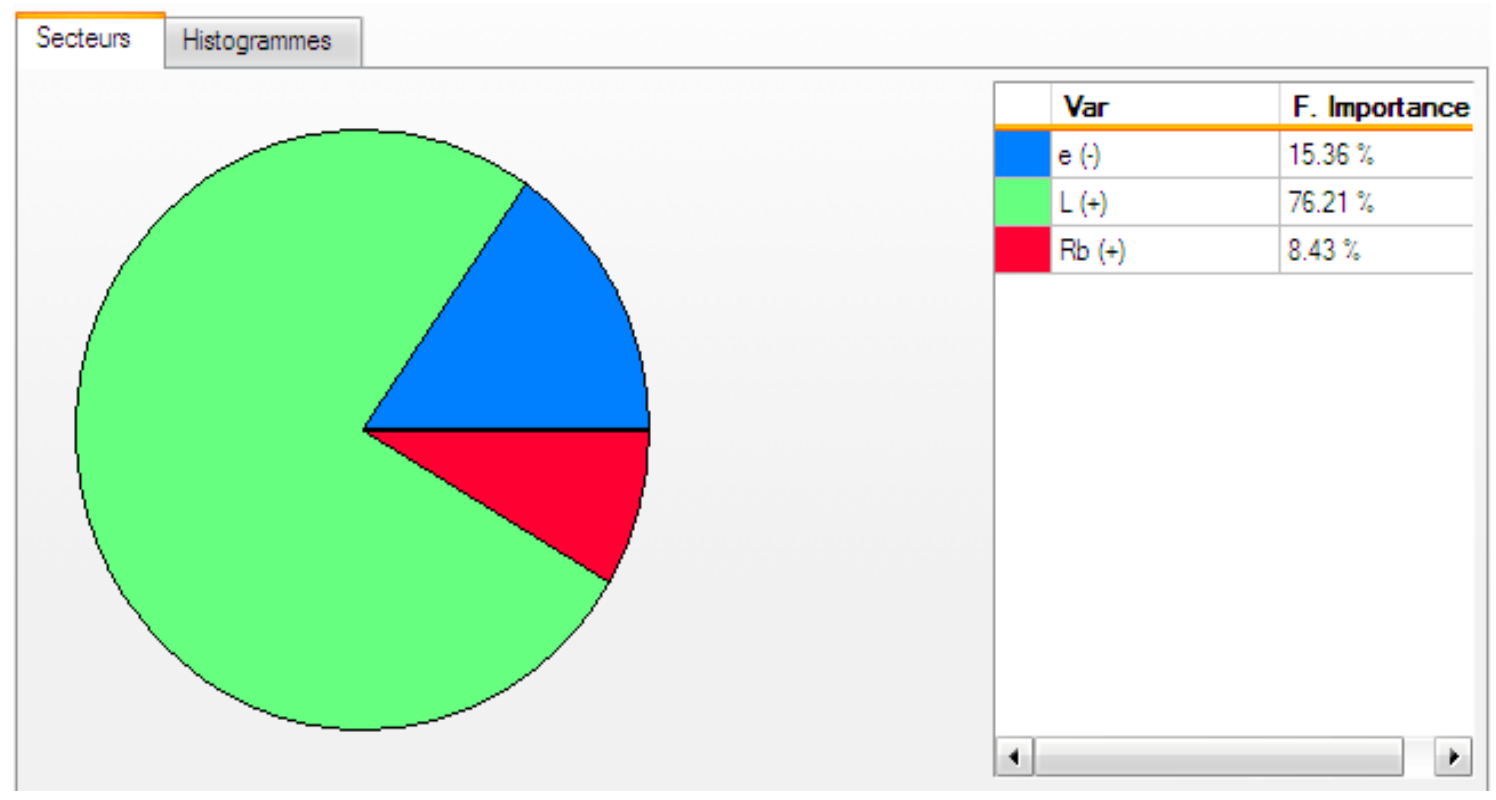

Figure 7. Important factors

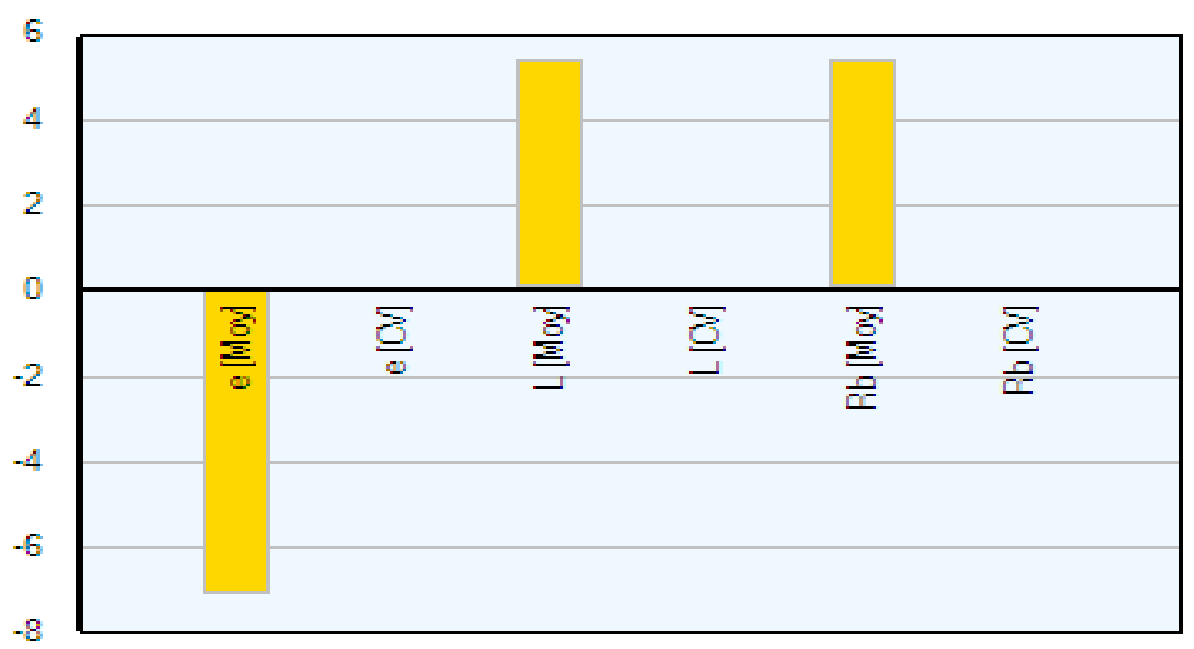

Figure 8. Diagram of elasticities 


\subsection{Form Inverse Method}

The inverse problem of FORM consists in giving the values of the desired Pf failure probability and then obtaining the corresponding mean L values. In this way, the designer can closely analyze the optimal designs for comparison and choice. The results obtained by this study are presented in the table below (Table 5).

Table 5. Results of the inverse problem.

\begin{tabular}{|c|c|c|c|}
\hline$\beta$ & $P_{f}$ & Average of $L(\mathrm{~mm})$ & Objective function \\
\hline 2 & 0.0228 & 25.9700 & 93.0903 \\
\hline 2.5 & $6.21 \times 10^{-3}$ & 26.0400 & 93.1463 \\
\hline 3 & $1.34 \times 10^{-3}$ & 26.1050 & 93.1983 \\
\hline 3.5 & $2.33 \times 10^{-4}$ & 26.1720 & 93.2519 \\
\hline 4 & $3.17 \times 10^{-5}$ & 26.2390 & 93.3055 \\
\hline 4.5 & $3.40 \times 10^{-6}$ & 26.3060 & 93.3591 \\
\hline 5 & $2.87 \times 10^{-7}$ & 26.3726 & 93.4124 \\
\hline 5.5 & $1.90 \times 10^{-8}$ & 26.4416 & 93.4676 \\
\hline 6 & $9.87 \times 10^{-10}$ & 26.5500 & 93.5543 \\
\hline
\end{tabular}

\section{Conclusions}

An optimization procedure based on deterministic approaches to a plate-terminated cam-rod mechanism has been studied in this work. The results of the study make it possible to select the geometrical parameters of the mechanism to obtain a minimum size and to ensure a good performance, while respecting the geometrical conditions, the constraints imposed on the performance and resistance indicators such as the pressure angle, the radius of curvature of the cam, and the contact pressure.

Subsequently, we made the reliability study of our system whose results are very encouraging. The calculation of the probability of system failure compared to the feared event was estimated by different methods, namely: FORM / SORM approximation methods and Monte Carlo simulations. Using approximation methods, we were able to obtain the sensitivity measures of this probability in relation to means and standard deviations as well as the elasticities of each random variable. The latter method can actually be a tool for design assistance or a tool for choosing the variables on which the optimal design of any mechanical system is based.

\section{REFERENCES}

[1] Rothbart HA (2004) Cam design handbook. McGraw-Hill, New Jersey.

[2] Yu Q, Lee HP (1998) Size optimization of cam mechanisms with translating roller followers. Proc Inst MechEng Part C
J MechEngSci 212: 381-386.

[3] Lampinen J (2003) Cam shape optimization by genetic algorithm. Comput Aided Des 35: 727-737.

[4] Hidalgo-Martinez M, Sanmiguel-Rojas E, Burgos MA (2014) Design of cams with negative radius follower using Bézier curves. Mech Mach Theory 82: 87-96.

[5] Hidalgo-Martinez M, Sanmiguel-Rojas E (2015) Minimization of the sliding velocity in planar cam mechanisms with flat-faced translating followers. Proc Inst MechEng Part C J MechEngSci 231(9):1632-1638.

[6] Flores P (2013) A computational approach for cam size optimization of disc cam follower mechanisms with translating roller followers. J Mech Rob 5(4):1-13.

[7] Mitsi S, Bouzakis KD, Tsiafis I, Mansour G ( 2001) Optimal synthesis of cam mechanism using cubic spline interpolation for cam NC milling. Journal of the Balkan Tribological Association 7(4): 225-233.

[8] Bouzakis KD, Mitsi S, Tsiafis I (1997) Computer aided optimum design and NC milling of planar cam mechanisms. Int J Mach Tool Manu 37(8): 1131-1142.

[9] Terauchi Y, El-Shakery SA (1983) A computer-aided method for optimum design of plate cam size avoiding undercutting and separation phenomena. Mech Mach Theory 18(2):157-163.

[10] Chan WH, Tseng CH, Tsay CB (1993) On the manufacturing tolerance of disk cam profile. J Mater Process Tech 38 (1-2):71-84.

[11] Chang WT, Wu LI (2009) Computerized tolerance analysis of disk cam mechanisms with a roller follower. EngComput 25 (3):247-2603.

[12] Phimeca engineer Software. http://www.phimeca.com

[13] Artobolevski L (1977) Théorie des mécanismes et des machines. Edition MIR, Moscou.

[14] Tsiafis I, Mitsi S, Bouzakis KD, Papadimitriou A (2013) Optimal design of a cam mechanism with translating flat-face follower using genetic algorithm. Tribology in industry 35(4): 255-260.

[15] Chen YF (1982) Mechanics and design of cam mechanisms. Pergamon Press, USA.

[16] Norton RL (2002) Cam Design and Manufacturing Handbook. Industrial Press, New York.

[17] Hamza F, Abderazek H, Lakhdar S, Ferhat D, Yıldız AR (2018) Optimum design of cam-roller follower mechanism using a new evolutionary algorithm. Int $\mathrm{J}$ Adv Manuf Techno 99(5-8): 1267-1282.

[18] Hammoudi A, Djeddou F, Atanasovska I (2017) Adaptive mixed differential evolution algorithm for bi-objective tooth profile spur gear optimization. Int J Adv Manuf Tech 90(5-8): 2063-2073.

[19] Zhang YM, Liu QL, Wen BC (2003) Practical reliability-based design of gear pairs. Mech Mach Theory 38(12): 1363-1370. 